PROCEEDINGS OF THE

AMERICAN MATHEMATICAL SOCIETY

Volume 134, Number 4, Pages 1031-1037

S 0002-9939(05)08143-8

Article electronically published on November 7, 2005

\title{
OBLIQUE PROJECTIONS AND FRAMES
}

\author{
J. ANTEZANA, G. CORACH, M. RUIZ, AND D. STOJANOFF
}

(Communicated by David R. Larson)

\begin{abstract}
We characterize those frames on a Hilbert space $\mathcal{H}$ which can be represented as the image of an orthonormal basis by an oblique projection defined on an extension $\mathcal{K}$ of $\mathcal{H}$. We show that all frames with infinite excess and frame bounds $1 \leq A \leq B$ are of this type. This gives a generalization of a result of Han and Larson which only holds for normalized tight frames.
\end{abstract}

\section{INTRODUCTION}

Let $\mathcal{H}$ be a (separable) Hilbert space, and let $\mathcal{F}=\left\{f_{n}\right\}_{n \in \mathbb{N}}$ be a sequence in $\mathcal{H}$. Then $\mathcal{F}$ is called a frame if there exist numbers $A, B>0$ such that, for every $f \in \mathcal{H}$,

$$
A\|f\|^{2} \leq \sum_{n \in \mathbb{N}}\left|\left\langle f, f_{n}\right\rangle\right|^{2} \leq B\|f\|^{2} .
$$

The optimal constants $A, B$ for equation (11) are called the frame bounds for $\mathcal{F}$. The frame $\mathcal{F}$ is called tight if $A=B$, and normalized tight if $A=B=1$.

Let $\mathcal{K}$ be another Hilbert space, and let $\mathcal{B}=\left\{e_{n}\right\}_{n \in \mathbb{N}}$ be an orthonormal basis of $\mathcal{K}$. It is known that, if $\mathcal{F}$ is a frame in $\mathcal{H}$, there exists a unique surjective bounded linear operator $T: \mathcal{K} \rightarrow \mathcal{H}$ such that $T e_{n}=f_{n}$, for all $n \in \mathbb{N}$. In this paper, the triple $(T, \mathcal{K}, \mathcal{B})$ is called a preframe operator for $\mathcal{F}$. The excess of $\mathcal{F}$ is the cardinal number $e(\mathcal{F})=\operatorname{dim} N(T)$, which does not depend on the chosen preframe operator.

A well-known theorem of Han and Larson [4] states that a sequence $\mathcal{F}=\left\{f_{n}\right\}_{n \in \mathbb{N}}$ in $\mathcal{H}$ is a normalized tight frame if and only if there exists an orthonormal basis $\mathcal{B}=\left\{e_{n}\right\}_{n \in \mathbb{N}}$ in an extension $\mathcal{K} \supseteq \mathcal{H}$ such that $f_{n}=P_{\mathcal{H}} e_{n}, n \in \mathbb{N}$, where $P_{\mathcal{H}} \in L(\mathcal{K})$ is the orthogonal projection onto $\mathcal{H}$, i.e., the triple $\left(P_{\mathcal{H}}, \mathcal{K}, \mathcal{B}\right)$ is a preframe operator for $\mathcal{F}$.

The main results of this paper extend their theorem as follows: A frame $\mathcal{F}=$ $\left\{f_{n}\right\}_{n \in \mathbb{N}}$ in $\mathcal{H}$ with frame bounds $1 \leq A \leq B$ comes from an orthonormal system, not necessarily complete, in an extension $\mathcal{K} \supseteq \mathcal{H}$, through a not necessarily orthogonal projection from $\mathcal{K}$ onto $\mathcal{H}$. If the excess of $\mathcal{F}$ is infinite, the orthonormal system can be chosen to be complete.

For frames with finite excess and frame bounds $1 \leq A \leq B$, there exist an orthonormal basis $\mathcal{B}=\left\{e_{n}\right\}_{n \in \mathbb{N}}$ of an extension $\mathcal{K} \supseteq \mathcal{H}$, and an oblique projection

Received by the editors September 22, 2004.

2000 Mathematics Subject Classification. Primary 42C15, 47A05.

Key words and phrases. Frames, oblique projections.

This research was partially supported by CONICET (PIP 2083/00), UBACYT I030, UNLP (11 X350) and ANPCYT (PICT03-9521).

(C)2005 American Mathematical Society 1031

Reverts to public domain 28 years from publication 
$Q \in L(\mathcal{K})$ onto $\mathcal{H}$ such that $Q e_{n}=f_{n}, n \in \mathbb{N}$, if and only if the rank of $T T^{*}-I$ is at most $e(\mathcal{F})$, where $(T, \mathcal{H}, \mathcal{E})$ is any preframe operator for $\mathcal{F}$ with domain $\mathcal{H}$. These results complete previous work by Casazza, Han and Larson [2].

In Section 2 we collect some preliminary facts. In Section 3, we present the mentioned extensions of the theorem of Han and Larson [4].

We thank Ole Christensen for his useful comments.

\section{Preliminaries}

Let $\mathcal{H}$ be a separable Hilbert space, and let $L(\mathcal{H})$ be the algebra of bounded linear operators on $\mathcal{H}$. For an operator $A \in L(\mathcal{H})$, we denote by $R(A)$ the range of $A$ and by $N(A)$ the nullspace of $A$.

Definition 2.1. The reduced minimum modulus $\gamma(T)$ of an operator $T \in L(\mathcal{H})$ is defined by

$$
\gamma(T)=\inf \left\{\|T x\|:\|x\|=1, x \in N(T)^{\perp}\right\} .
$$

It is well known that $\gamma(T)=\gamma\left(T^{*}\right)=\gamma\left(T^{*} T\right)^{1 / 2}$. Also, it can be shown that an operator $T$ has closed range if and only if $\gamma(T)>0$. In this case, $\gamma(T)=$ $\left\|T^{\dagger}\right\|^{-1}$.

Frames. We introduce some basic facts about frames in Hilbert spaces. For complete descriptions of frame theory and applications, the reader is referred to the survey by Heil and Walnut [5] or the books by Young [7] and Christensen [3].

Definition 2.2. Let $\mathcal{H}$ be a separable Hilbert space, and let $\mathcal{F}=\left\{f_{n}\right\}_{n \in \mathbb{N}}$ be a sequence in $\mathcal{H}$.

i. $\mathcal{F}$ is called a frame if there exist numbers $A, B>0$ such that, for every $f \in \mathcal{H}$,

$$
A\|f\|^{2} \leq \sum_{n \in \mathbb{N}}\left|\left\langle f, f_{n}\right\rangle\right|^{2} \leq B\|f\|^{2} .
$$

ii. The optimal constants $A, B$ for equation (3) are called the frame bounds for $\mathcal{F}$.

iii. The frame $\mathcal{F}$ is called tight if $A=B$, and normalized tight if $A=B=1$.

Definition 2.3. Let $\mathcal{F}=\left\{f_{n}\right\}_{n \in \mathbb{N}}$ be a frame in $\mathcal{H}$. Let $\mathcal{K}$ and $\mathcal{H}^{\prime}$ be separable Hilbert spaces such that $\mathcal{H}$ is a closed subspace of $\mathcal{H}^{\prime}$. Let $\mathcal{B}=\left\{\varphi_{n}: n \in \mathbb{N}\right\}$ be an orthonormal basis of $\mathcal{K}$. From equation (3), it follows that there exists a unique $T \in L\left(\mathcal{K}, \mathcal{H}^{\prime}\right)$ such that

$$
T\left(\varphi_{n}\right)=f_{n}, \quad n \in \mathbb{N} .
$$

We shall say that the triple $(T, \mathcal{K}, \mathcal{B})$ is a preframe (or synthesis) operator for $\mathcal{F}$. Another consequence of equation (3) is that $R(T)=\mathcal{H}$. In particular, if $\mathcal{H}^{\prime}=\mathcal{H}$, then $T$ is surjective.

Remark 2.4. Let $\mathcal{F}=\left\{f_{n}\right\}_{n \in \mathbb{N}}$ be a frame in $\mathcal{H}$, and let $(T, \mathcal{K}, \mathcal{B})$ be a preframe operator for $\mathcal{F}$.

i. The frame bounds of $\mathcal{F}$ can be computed in terms of the preframe operator:

$$
A=\gamma(T)^{2} \quad \text { and } \quad B=\|T\|^{2} .
$$

ii. The adjoint $T^{*} \in L\left(\mathcal{H}^{\prime}, \mathcal{K}\right)$ of $T$ is given by $T^{*}(x)=\sum_{n \in \mathbb{N}}\left\langle x, f_{n}\right\rangle \varphi_{n}, x \in \mathcal{H}$. It is called an analysis operator for $\mathcal{F}$. 
iii. If $\left(T_{1}, \mathcal{K}_{1}, \mathcal{B}_{1}\right)$ is another preframe operator for $\mathcal{F}$, then there exists a unique unitary operator $U \in L\left(\mathcal{K}_{1}, \mathcal{K}\right)$ which sends $\mathcal{B}_{1}$ onto $\mathcal{B}$, and therefore $T_{1}=$ $T U$. It follows that $S=T T^{*}=T_{1} T_{1}^{*}$; then $S f=\sum_{n \in \mathbb{N}}\left\langle f, f_{n}\right\rangle f_{n}, f \in \mathcal{H}$. If we consider $S$ as acting on $\mathcal{H}$, it follows from (3) that $A . I \leq S \leq B . I$, so that $S$ is a positive invertible operator on $\mathcal{H}$. Moreover, the optimal constants $A, B$ for equation (3) are

$$
B=\|S\|=\rho(S) \text { and } A=\gamma(S)=\left\|S^{-1}\right\|^{-1}=\min \{\lambda: \lambda \in \sigma(S)\} .
$$

$S$ is called the frame operator of $\mathcal{F}$. Note that by the previous observation the frame operator does not depend on the chosen preframe operator. Hence, $\mathcal{F}$ is tight if and only if $S=A I$ and normalized tight if and only if $S=I$.

Definition 2.5. Let $\mathcal{F}=\left\{f_{n}\right\}_{n \in \mathbb{N}}$ be a frame in $\mathcal{H}$, and let $(T, \mathcal{K}, \mathcal{B})$ be a preframe operator for $\mathcal{F}$. The cardinal number

$$
e(\mathcal{F})=\operatorname{dim} N(T)
$$

is called the excess of the frame. Note that by Remark $2.4 e(\mathcal{F})$ does not depend on the chosen preframe operator. In particular,

$$
e(\mathcal{F})=\operatorname{dim}\left\{\left(c_{n}\right)_{n \in \mathbb{N}} \in \ell^{2}: \sum_{n \in \mathbb{N}} c_{n} f_{n}=0\right\},
$$

which is the nullity of the preframe operator induced by the canonical basis of $\ell^{2}$. Holub [6] and Balan, Casazza, Heil and Landau [1] proved that

$$
e(\mathcal{F})=\sup \left\{|I|: I \subseteq \mathbb{N} \text { and }\left\{f_{n}\right\}_{n \notin I} \text { is still a frame on } \mathcal{H}\right\} .
$$

This characterization justifies the name "excess of $\mathcal{F}$ ". The frame $\mathcal{F}$ is called a Riesz basis if $e(\mathcal{F})=0$, i.e., if $\mathcal{F}$ is the image of an orthonormal basis of $\mathcal{K}$ by an isomorphism $T \in L(\mathcal{K}, \mathcal{H})$.

\section{Projections AND FRAMES}

Frames and projections are related in a variety of ways. For instance, since a frame $\mathcal{F}$ for $\mathcal{H}$ admits a preframe operator $(T, \mathcal{K}, \mathcal{B})$ and $T \in L(\mathcal{K})$ is surjective with range $\mathcal{H}$, any right inverse $U$ of $T$ provides a projection $U T \in L(\mathcal{K})$. In this paper we study another type of relationship between frames and projections. Namely, we consider an extension of a theorem by Han and Larson 4. Along this section we use the fact that every subspace $\mathcal{S}$ of $\mathcal{H}$ induces a representation of elements of $L(\mathcal{H})$ by $2 \times 2$ block matrices; that is, we shall identify each $A \in L(\mathcal{H})$ with a $2 \times 2$ matrix $\left(\begin{array}{ll}A_{11} & A_{12} \\ A_{21} & A_{22}\end{array}\right) \quad \begin{aligned} & \mathcal{S} \\ & \mathcal{S}^{\perp}\end{aligned}$, which we write to emphasize the decomposition which induces it. For example, it is easy to see that $Q \in L(\mathcal{H})$ is an oblique projection with $R(Q)=\mathcal{S}$ if and only if $Q$ has matrix form $Q=\left(\begin{array}{cc}I & X \\ 0 & 0\end{array}\right) \mathcal{S}_{\mathcal{S}^{\perp}}^{\mathcal{S}}$, for some $X \in L\left(\mathcal{S}^{\perp}, \mathcal{S}\right)$.

In 4, Han and Larson characterized normalized tight frames as follows.

Theorem 3.1 (Han and Larson). A sequence $\mathcal{F}=\left\{f_{n}\right\}_{n \in \mathbb{N}}$ in a Hilbert space $\mathcal{H}$ is a normalized tight frame if and only if there exist a Hilbert space $\mathcal{K} \supseteq \mathcal{H}$ and an orthonormal basis $\mathcal{B}=\left\{e_{n}\right\}_{n \in \mathbb{N}}$ of $\mathcal{K}$ such that the triple $\left(P_{\mathcal{H}}, \mathcal{K}, \mathcal{B}\right)$ is a preframe operator for $\mathcal{F}$, i.e., $f_{n}=P_{\mathcal{H}} e_{n}, n \in \mathbb{N}$. 
In what follows we shall study the following question: given a frame $\mathcal{F}=\left\{f_{n}\right\}_{n \in \mathbb{N}}$ in $\mathcal{H}$, does there exist a Hilbert space $\mathcal{K} \supseteq \mathcal{H}$, an orthonormal basis $\left\{e_{n}\right\}_{n \in \mathbb{N}}$ of $\mathcal{K}$ and an oblique (i.e., not necessarily orthogonal) projection $Q \in L(\mathcal{K})$ such that $Q e_{n}=f_{n}, n \in \mathbb{N}$ ? In other words, does a preframe operator $(Q, \mathcal{K}, \mathcal{B})$ exist for $\mathcal{F}$ such that $Q$ is idempotent?

This is not true in general. Indeed, there exist at least two obstructions:

i. Every oblique projection $Q$ satisfies that $\gamma(Q) \geq 1$, because

$$
Q Q^{*} \geq Q P_{R(Q)} Q^{*}=P_{R(Q)}, R\left(Q Q^{*}\right)=R(Q), \quad \text { and } \gamma(P)=1
$$

for every orthogonal projection $P$. Therefore, if there exists a representation of a frame $\mathcal{F}$ as above, then its frame bounds must satisfy $1 \leq A \leq B$. This obstruction is not essential because it can be fixed by multiplying the frame by a convenient positive constant.

ii. Even if the frame bounds of $\mathcal{F}$ satisfy $1 \leq A \leq B$, the representation may not exist if $e(\mathcal{F})$ is finite. For example, suppose that $\mathcal{F}$ is a Riesz basis and $f_{n}=Q e_{n}$ for an oblique projection $Q \in L(\mathcal{K})$ and an orthonormal basis $\mathcal{B}=\left\{e_{n}\right\}_{n \in \mathbb{N}}$ of $\mathcal{K} \supseteq \mathcal{H}$. Then, since $(Q, \mathcal{K}, \mathcal{B})$ is a preframe operator for $\mathcal{F}$, it holds that $\operatorname{dim} N(Q)=e(\mathcal{F})=0$. Thus, $\mathcal{K}=\mathcal{H}, Q=I$ and $\mathcal{F}=\mathcal{B}$. This means that orthonormal bases are the only Riesz bases which admit such a representation.

The paper 2] has a section addressed to this problem. There is, however, an inaccuracy in the statement of its Theorem 3.2. Indeed, it states that every frame for $\mathcal{H}$ can be obtained as the image of an orthonormal basis of an extension of $\mathcal{H}$ by an oblique projection. The next theorems complete, in some sense, the results of Casazza, Han and Larson [2], section 3.

In the rest of this section we shall show that the mentioned representation can be obtained for frames which satisfy the frame bounds restrictions and which have infinite excess. For frames with finite excess (and good frame bounds), the representation can be obtained by considering orthonormal systems instead of orthonormal bases. We also give equivalent conditions on a frame with finite excess in order to admit such a representation with an orthonormal basis.

Theorem 3.2. Let $\mathcal{F}=\left\{f_{n}\right\}_{n \in \mathbb{N}}$ be a frame in an infinite dimensional Hilbert space $\mathcal{H}$, with frame bounds $1 \leq A \leq B$. Denote $\mathcal{K}=\mathcal{H} \oplus \mathcal{H}$. Then there exist an oblique projection $Q \in L(\mathcal{K})$ with $R(Q)=\mathcal{H} \oplus\{0\}$ and an orthonormal system $\left\{b_{n}\right\}_{n \in \mathbb{N}}$ in $\mathcal{K}$, such that

$$
f_{n} \oplus 0=Q b_{n}, \quad n \in \mathbb{N} .
$$

Moreover, if $e(\mathcal{F})=\infty$, then the sequence $\left\{b_{n}\right\}_{n \in \mathbb{N}}$ can be supposed to be an orthonormal basis of $\mathcal{K}$, i.e., $\left(Q, \mathcal{K},\left\{b_{n}\right\}_{n \in \mathbb{N}}\right)$ is a preframe operator for $\mathcal{F}$.

Proof. Let $(T, \mathcal{H}, \mathcal{B})$ be a preframe operator for $\mathcal{F}$, with $T \in L(\mathcal{H})$. By hypothesis, $T T^{*} \geq A I \geq I$. Denote by $X=\left(T T^{*}-I\right)^{1 / 2} \in L(\mathcal{H})^{+}$. We shall identify $\mathcal{H}$ with $\overline{\mathcal{H}} \oplus\{0\}$, i.e., we identify $f_{n} \sim f_{n} \oplus 0 \in \mathcal{K}$. Let $\tilde{T}: \mathcal{H} \rightarrow \mathcal{K}$ be defined by $\tilde{T} x=T x \oplus 0$. Then $\tilde{T} \tilde{T}^{*} \sim\left(\begin{array}{cc}T T^{*} & 0 \\ 0 & 0\end{array}\right) \in L(\mathcal{K})$. Let

$$
Q=\left(\begin{array}{cc}
I_{\mathcal{H}} & X \\
0 & 0
\end{array}\right) \begin{aligned}
& \mathcal{H} \\
& \mathcal{H}
\end{aligned} \in L(\mathcal{K})
$$


Then it is clear that $Q$ is a projection on $\mathcal{K}$ with $R(Q)=\mathcal{H} \oplus 0$. Moreover,

$$
Q Q^{*}=\left(\begin{array}{cc}
I_{\mathcal{H}}+X X^{*} & 0 \\
0 & 0
\end{array}\right)=\tilde{T} \tilde{T}^{*}
$$

i.e., $\left|Q^{*}\right|=\left|\tilde{T}^{*}\right|$.

Consider the (right) polar decomposition $T=\left|T^{*}\right| V ; V \in L(\mathcal{H})$ is a partial isometry with initial space $N(T)^{\perp}$ and final space $R(T)=\mathcal{H}$. Define $U: \mathcal{H} \rightarrow \mathcal{K}$ by

$$
U x=V P_{N(T)}^{\perp} x \oplus P_{N(T)} x, \quad x \in \mathcal{H} .
$$

Then, $U$ is an isometry and $\tilde{T}=\left|\tilde{T}^{*}\right| U$. The partial isometry of the right polar decomposition of $Q$ extends to a unitary operator $W$ on $\mathcal{K}$, because $\operatorname{dim}(N(Q))=$ $\operatorname{dim}\left(R(Q)^{\perp}\right)$. Moreover, $Q=\left|Q^{*}\right| W$. Then

$$
\tilde{T}=\left|\tilde{T}^{*}\right| U=\left|Q^{*}\right| U=Q W^{*} U .
$$

Therefore, if $\mathcal{B}=\left\{e_{n}\right\}_{n \in \mathbb{N}}$,

$$
f_{n}=T e_{n} \sim T e_{n} \oplus 0=\tilde{T} e_{n}=Q\left(W^{*} U e_{n}\right)=Q b_{n}, \quad n \in \mathbb{N},
$$

where $\left\{b_{n}\right\}_{n \in \mathbb{N}}=\left\{W^{*} U e_{n}\right\}_{n \in \mathbb{N}}$, which is clearly an orthonormal system in $\mathcal{K}$.

Suppose now that $e(\mathcal{F})=\operatorname{dim} N(T)=\infty$. We shall show that the isometry $U$ defined in equation (5) can be changed to a unitary operator from $\mathcal{H}$ onto $\mathcal{K}$, still satisfying that $\tilde{T}=\left|\tilde{T}^{*}\right| U$. In order to do this, take

$$
U x=V P_{N(T)} \perp x \oplus Y P_{N(T)} x, \quad x \in \mathcal{H},
$$

where $Y \in L(\mathcal{H})$ is a partial isometry with initial space $N(T)$ and final space $\mathcal{H}$. It follows that $U$ isometrically maps $N(T)^{\perp}$ onto $\mathcal{H} \oplus\{0\}$ and $N(T)$ onto $\{0\} \oplus \mathcal{H}$. Then the sequence $b_{n}=W^{*} U e_{n}, n \in \mathbb{N}$, turns out to be an orthonormal basis of $\mathcal{K}$.

Remark 3.3. Using the notation of Theorem 3.2. if $\mathcal{K}_{0}=\overline{\operatorname{span}}\left\{b_{n}\right\}$ and $Q_{0}=\left.Q\right|_{\mathcal{K}_{0}}$, then it might be possible to consider orthonormal bases instead of orthonormal systems. Nevertheless, Theorem 3.4 below shows that this argument fails, in general. In fact, $\mathcal{H}$ is not necessarily contained in $\mathcal{K}_{0}$, in which case $Q_{0}$ is not a projection.

In the case that $e(\mathcal{F})<\infty$, we have the following theorem.

Theorem 3.4. Let $\mathcal{F}=\left\{f_{n}\right\}_{n \in \mathbb{N}}$ be a frame in $\mathcal{H}$ with frame bounds $1 \leq A \leq B$. Suppose that $e(\mathcal{F})<\infty$. Then, the following conditions are equivalent:

(1) There exist a Hilbert space $\mathcal{K}$ such that $\mathcal{H} \subseteq \mathcal{K}$, a projection $Q \in L(\mathcal{K})$, and an orthonormal basis $\mathcal{B}=\left\{b_{n}\right\}_{n \in \mathbb{N}}$ of $\mathcal{K}$ such that $f_{n}=Q b_{n}$ for all $n \in \mathbb{N}$.

(2) If $(T, \mathcal{L}, \mathcal{E})$ is a preframe operator for $\mathcal{F}$, then $\operatorname{dim} R\left(T T^{*}-I_{\mathcal{H}}\right) \leq e(\mathcal{F})$.

Proof. Recall that the frame operator $T T^{*}$ of $\mathcal{F}$ does not depend on the preframe $(T, \mathcal{L}, \mathcal{E})$ chosen. We shall consider a preframe $(T, \mathcal{H}, \mathcal{E})$ for some orthonormal basis $\mathcal{E}$ of $\mathcal{H}$. If the first condition holds, then $(Q, \mathcal{K}, \mathcal{B})$ is another preframe operator for $\mathcal{F}$. Therefore $e(\mathcal{F})=\operatorname{dim} N(T)=\operatorname{dim} N(Q)=\operatorname{dim}(\mathcal{K} \ominus \mathcal{H})$. We shall identify $\mathcal{K} \ominus \mathcal{H}$ with $N(T)$, and so $\mathcal{K}$ with $\mathcal{H} \oplus N(T)$. We shall also identify $\mathcal{H}$ with $\mathcal{H} \oplus\{0\}$, in particular, $f_{n} \sim f_{n} \oplus 0 \in \mathcal{K}$. Let $\tilde{T}: \mathcal{H} \rightarrow \mathcal{K}$, given by $\tilde{T} x=T x \oplus 0$. 


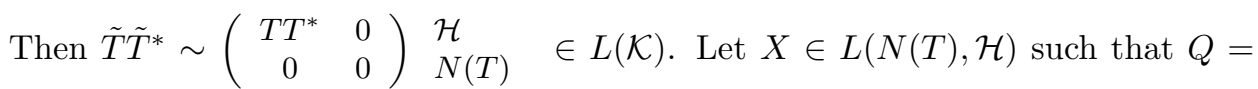

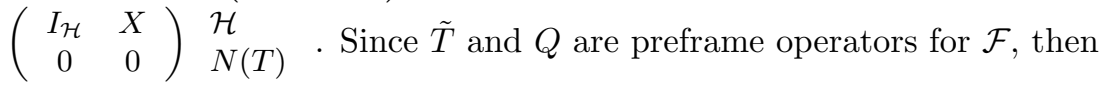

$$
\tilde{T} \tilde{T}^{*}=Q Q^{*}=\left(\begin{array}{cc}
I_{\mathcal{H}}+X X^{*} & 0 \\
0 & 0
\end{array}\right) .
$$

Thus, $T T^{*}-I_{\mathcal{H}}=X X^{*}$, while $\operatorname{dim} R\left(X X^{*}\right) \leq \operatorname{dim} R(X) \leq \operatorname{dim} N(T)$.

Conversely, if $\operatorname{dim} R\left(T T^{*}-I\right) \leq \operatorname{dim} N(T)$, we consider, as in the proof of Theorem 3.2, $X=\left(T T^{*}-I\right)^{1 / 2} \in L(\mathcal{H})^{+}$. Note that $\operatorname{dim} N(X)^{\perp}=\operatorname{dim} R(X) \leq$ $\operatorname{dim} N(T)$. Then there exists a partial isometry $V: N(T) \rightarrow \mathcal{H}$ with $V V^{*}=$ $P_{N(X)^{\perp}}$. As before, we use the Hilbert space $\mathcal{K}=\mathcal{H} \oplus N(T)$, and the operator $\tilde{T} \in L(\mathcal{H}, \mathcal{K})$, given by $\tilde{T} x=T x \oplus 0$. Let $Y=X V \in L(N(T), \mathcal{H})$, and

$$
Q=\left(\begin{array}{cc}
I_{\mathcal{H}} & Y \\
0 & 0
\end{array}\right) \begin{aligned}
& \mathcal{H} \\
& N(T)
\end{aligned} \in L(\mathcal{K})
$$

Then $Q^{2}=Q, R(Q)=\mathcal{H} \oplus\{0\}$ and $\left|Q^{*}\right|=\left|\tilde{T}^{*}\right|$, because $Y Y^{*}=X V V^{*} X^{*}=$ $X P_{N(X) \perp} X^{*}=X X^{*}$, so that

$$
Q Q^{*}=\left(\begin{array}{cc}
I+Y Y^{*} & 0 \\
0 & 0
\end{array}\right)=\left(\begin{array}{cc}
I+X X^{*} & 0 \\
0 & 0
\end{array}\right)=\tilde{T} \tilde{T}^{*} .
$$

The rest of the proof follows the same lines as the first part of the proof of Theorem 3.2, but taking $\mathcal{K}=\mathcal{H} \oplus N(T)$. Note that, in this case, the isometry $U$ defined in equation (5) becomes a unitary operator from $\mathcal{H}$ onto $\mathcal{K}$. Then, if $W$ is the unitary operator considered in the first part of the proof of Theorem 3.2. the sequence $b_{n}=W^{*} U e_{n}, n \in \mathbb{N}$, becomes an orthonormal basis of $\mathcal{K}$.

Remark 3.5. Theorem 3.1 can also be generalized by replacing the orthonormal basis $\left\{e_{k}\right\}_{k \in \mathbb{N}}$ by a Riesz basis in $\mathcal{K}$; in this way, we get arbitrary (i.e., not necessarily normalized tight) frames. This result was proved by Han and Larson 4, Proposition 1.6, but with a different formulation. The translation to this setting is the following:

A sequence $\mathcal{F}=\left\{f_{n}\right\}_{n \in \mathbb{N}}$ in a Hilbert space $\mathcal{H}$ is a frame if and only if there exist a Hilbert space $\mathcal{K} \supseteq \mathcal{H}$ and a Riesz basis $\left\{x_{k}\right\}_{k \geq 1}$ of $\mathcal{K}$ such that $f_{n}=P_{\mathcal{H}} x_{n}$, $n \in \mathbb{N}$. Moreover, the Riesz basis can be chosen in such a way that it has the same lower frame bound as $\mathcal{F}$, and the sequence $\left(I-P_{\mathcal{H}}\right) x_{n}, n \in \mathbb{N}$, is a normalized tight frame for $\mathcal{K} \ominus \mathcal{H}$.

We give an alternative proof for completeness: Suppose that $\mathcal{F}$ is a frame. Let $(T, \mathcal{H}, \mathcal{B})$ be a preframe operator for $\mathcal{F}$. Let $\mathcal{K}=\mathcal{H} \oplus N(T)$. We shall identify $\mathcal{H}$ with $\mathcal{H} \oplus\{0\} \subseteq \mathcal{K}$, writing $x \sim x \oplus 0$, for $x \in \mathcal{H}$. Let $V: \mathcal{H} \mapsto \mathcal{K}$ be defined by:

$$
V x=T x \oplus P_{N(T)} x=T P_{N(T)^{\perp}} x \oplus P_{N(T)} x, \quad x \in \mathcal{H} .
$$

Since $T\left(N(T)^{\perp}\right)=R(T)=\mathcal{H}$, it follows that $V$ is bounded, linear and bijective. Hence, if $\mathcal{B}=\left\{e_{n}\right\}_{n \in \mathbb{N}}$, then $x_{n}=V e_{n}$ is a Riesz basis in $\mathcal{K}$. Note that $f_{n} \sim$ $f_{n} \oplus 0=P_{\mathcal{H}} x_{n}, n \in \mathbb{N}$. By Theorem 3.1, the sequence $\left(I-P_{\mathcal{H}}\right) x_{n}=P_{N(T)} e_{n}$, $n \in \mathbb{N}$, is a normalized tight frame for $N(T)=\mathcal{K} \ominus \mathcal{H}$. Finally, $\gamma(V)=\gamma(T)$ and $\|V\|=\max \{1,\|T\|\}$. Therefore, the optimal lower frame bound of $\left\{x_{n}\right\}_{n \in \mathbb{N}}$ is the same as those of $\mathcal{F}$. The converse is clear. 


\section{REFERENCES}

[1] R. Balan, P. Casazza, C. Heil and Z. Landau, Deficits and excesses of frames, Advances in Computational Mathematics 18 (2003), 93-116. MR.1968114 (2004a:42040)

[2] P. G. Casazza, D. Han and D. R. Larson, Frames for Banach spaces, Contemp. Math. 247 (1999), 149-182. MR 1738089 (2000m:46015)

[3] O. Christensen, An introduction to frames and Riesz bases, Birkhäuser, Boston, 2003. MR:1946982 (2003k:42001)

[4] D. Han and D. R. Larson, Frames, bases and group representations, Mem. Amer. Math. Soc. 147 (2000), no. 697. MR.1686653 (2001a:47013)

[5] C. E. Heil and D. F. Walnut, Continuous and discrete wavelet transforms, SIAM Rev. 31 (1989), 628-666. MR1025485 (91c:42032)

[6] J. R. Holub, Pre-frame operators, Besselian frames and near-Riesz bases in Hilbert spaces, Proc. Amer. Math. Soc. 122 (1994), 779-785. MR1204376 (95a:46030)

[7] R. M. Young, An introduction to nonharmonic Fourier series (revised first edition), Academic Press, San Diego, 2001. MR1836633 (2002b:42001)

iam-Conicet and Departamento de Matemática, FCE-UnlP, La Plata, Argentina E-mail address: antezana@mate.unlp.edu.ar

iam-Conicet and Departamento de Matemática, Fi-UBA, Saavedra 15, Piso 3 (1083), Ciudad Autónoma de Buenos Aires, Argentina

E-mail address: gcorach@fi.uba.ar

iAm-Conicet and Departamento de Matemática, FCE-Unlp, La Plata, Argentina E-mail address: mruiz@mate.unlp.edu.ar

iam-Conicet and Departamento de Matemática, FCE-UnlP, La Plata, Argentina E-mail address: demetrio@mate.unlp.edu.ar 